\title{
ERRATUM
}

\section{Quantitative Relationships Between Structure and Pharmacokinetics of Beta-Adrenoceptor Blocking Agents in Man ${ }^{1}$}

\author{
Peter H. Hinderling, ${ }^{2}$ Olga Schmidlin, ${ }^{2,4}$ and Jochen K. Seydel ${ }^{3}$
}

A review of the steady state volume of distribution $\left(V_{S S}\right)$ data of the beta-adrenoceptor blocking agents (BA) in the report (Vol. 12, No. 3, p. 272 , Table II) showed that an erroneous value of $37 l$ had been calculated and employed for timolol. As this value of $V_{S S}$ was used in calculating the steady state volume of distribution referenced to the unbound drug in plasma, $V u_{S S}$, the ratio of the fraction of drug bound and unbound to tissue, $r_{T}$, and the mean residence time, $M R T$, the values of these three parameters were also erroneous for timolol. Consequently, the regressions (linear or parabolic) of $V u_{S S}, r_{T}$, and $M R T$ against the apparent octanol/buffer $\left(p H\right.$ 7.4) partition coefficient determined by the shake flask method, $K_{S F}^{17.4}$, and against the partition coefficient of drug between plasma and plasma water, $K p$, had to be repeated for the whole series of BA tested. On the basis of the new regression equations obtained, the predictions of the $V u_{S S^{-}}$ and $M R T$-values of the BA considered were then revised.

The correct values for $V_{S S}(100 l), V u_{S S}(270 l), r_{T}(0.840)$, and $M R T$ $(168 \mathrm{~min})$ for timolol are given in the revised Tables II and III. The correct coefficients of the $\log -\log$ regressions of $V u_{S S}, r_{T}$, and $M R T$ on $K_{S F}^{\prime 7.4}$ and $K p$ for the BA tested are presented in Tables IV and V, respectively. The $\log -\log$ fits of the recalculated $V u_{S S^{-}}$and $M R T$-values against $K_{S F}^{\prime 7.4}$ are shown in Figs. 4 and 8, and the correctly predicted values for $V u_{S S}$ and

\footnotetext{
'J. Pharmacokin. Biopharm. 12: 263-287 (1984).

${ }^{2}$ Department of Pharmacology, Biocenter, University of Basle, Klingelbergstr. 70, 4056 Basle, Switzerland.

${ }^{3}$ Department of Medicinal and Pharmaceutical Chemistry, Research Institute Borstel, Parkallee 1-40, 2061 Borstel, Federal Republic of Germany,

${ }^{4}$ Present Address: Department of Internal Medicine, University of Basle, Petersgraben 6, 4059 Basle, Switzerland.
} 


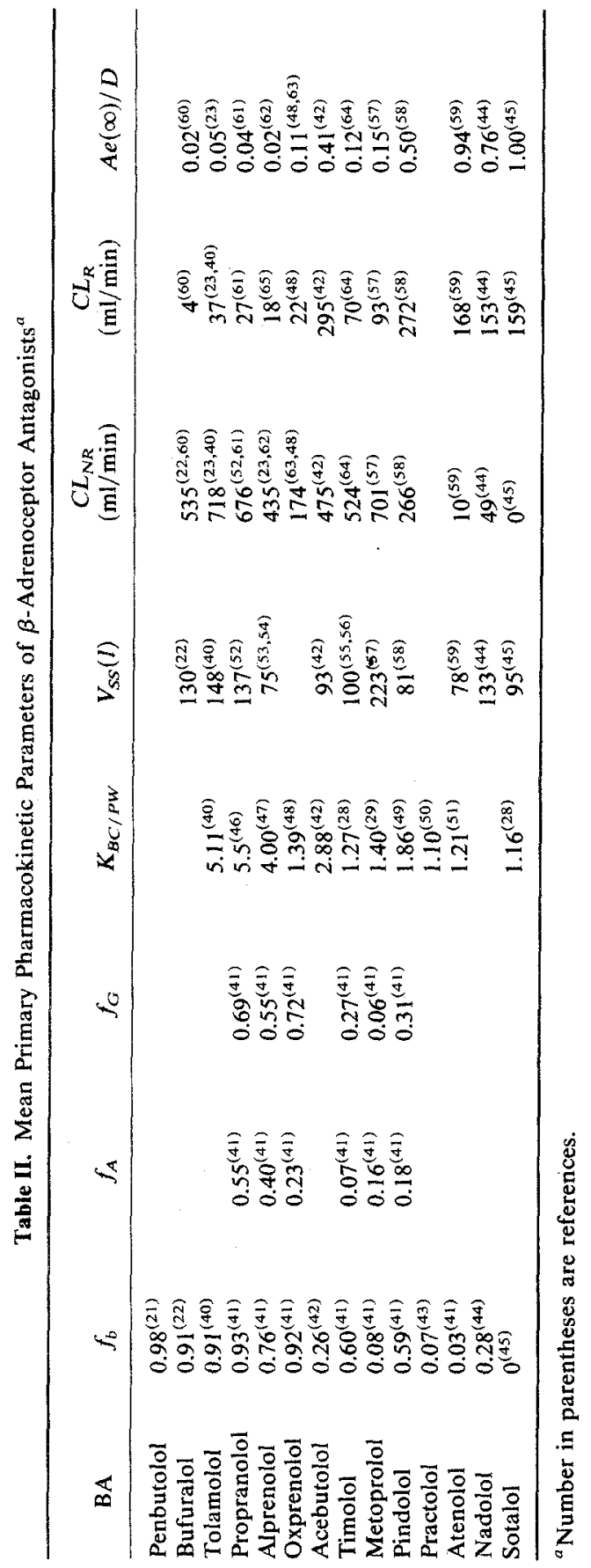




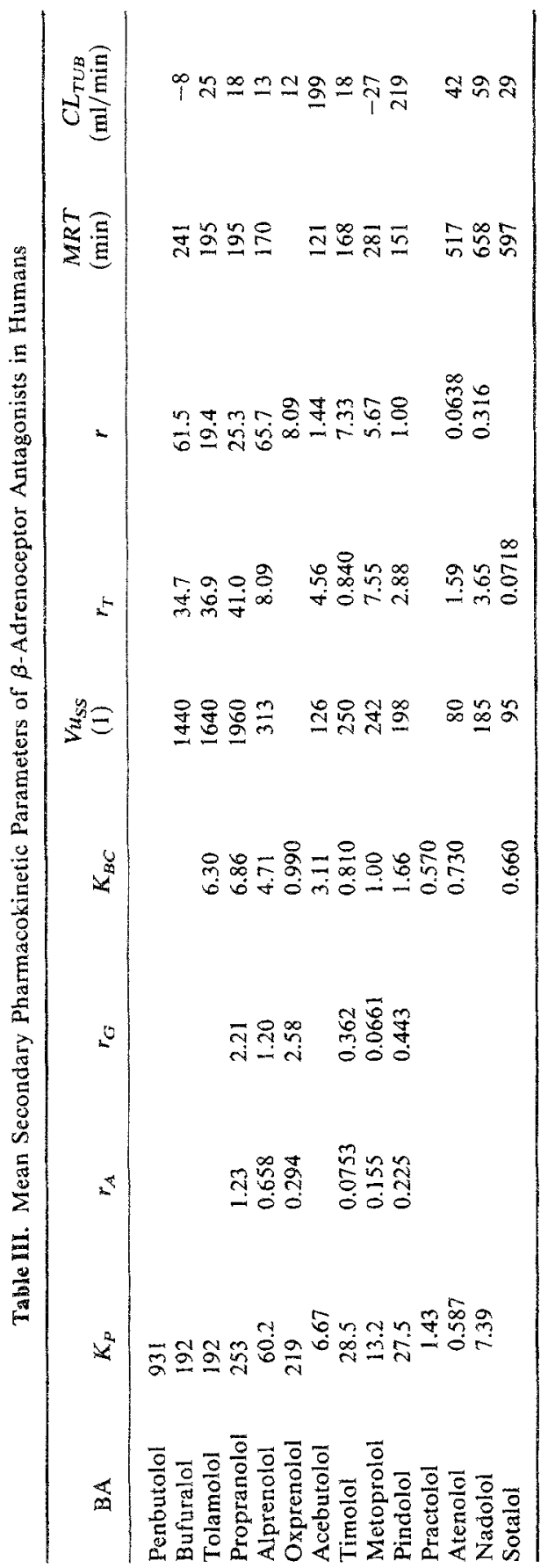




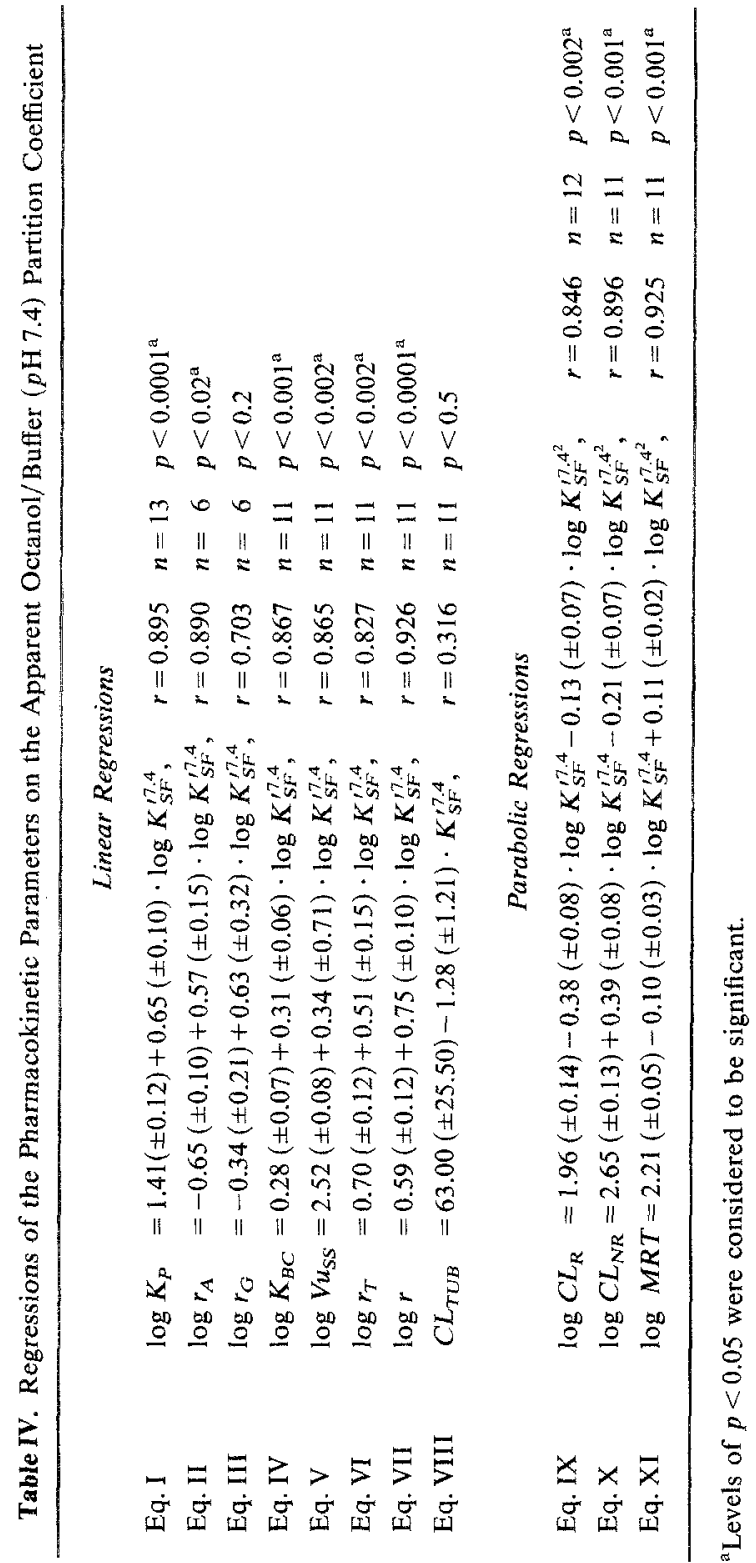




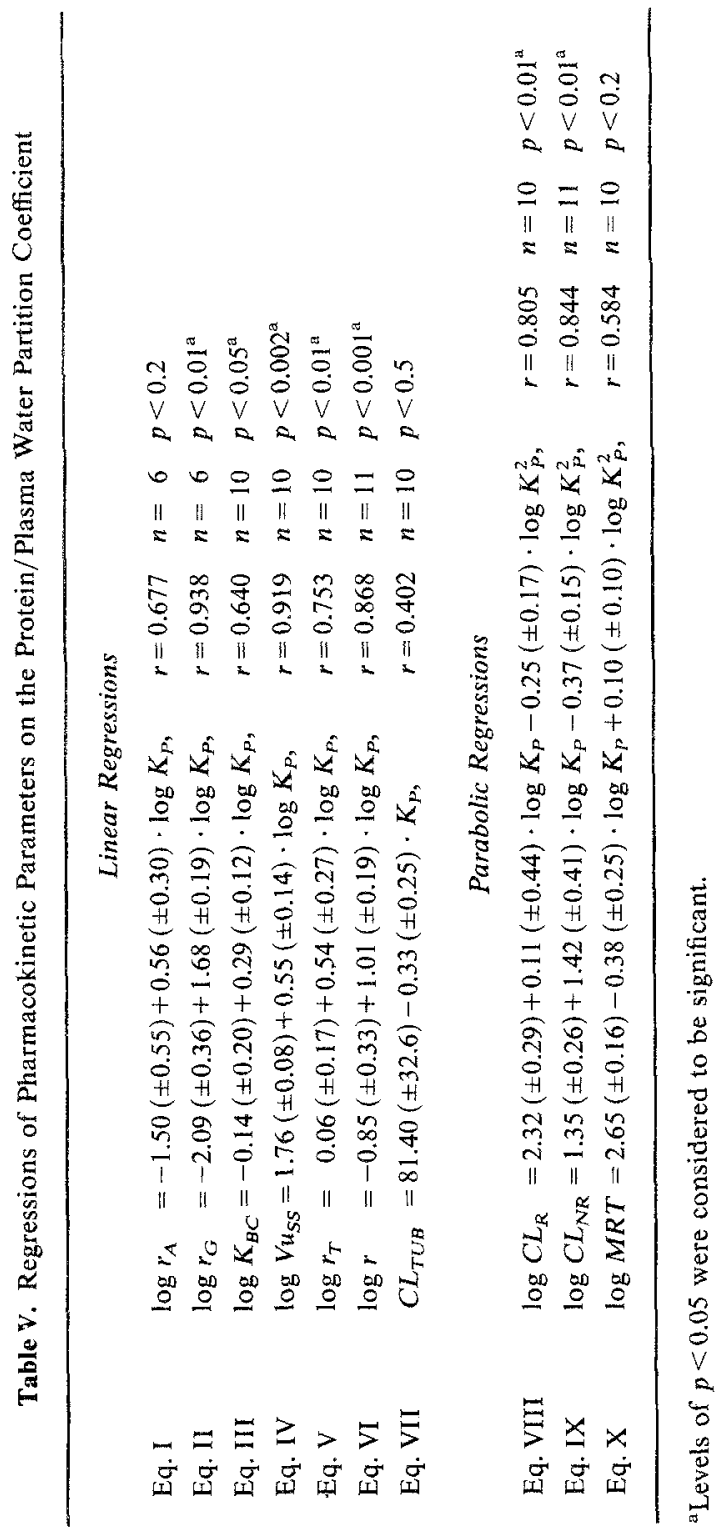




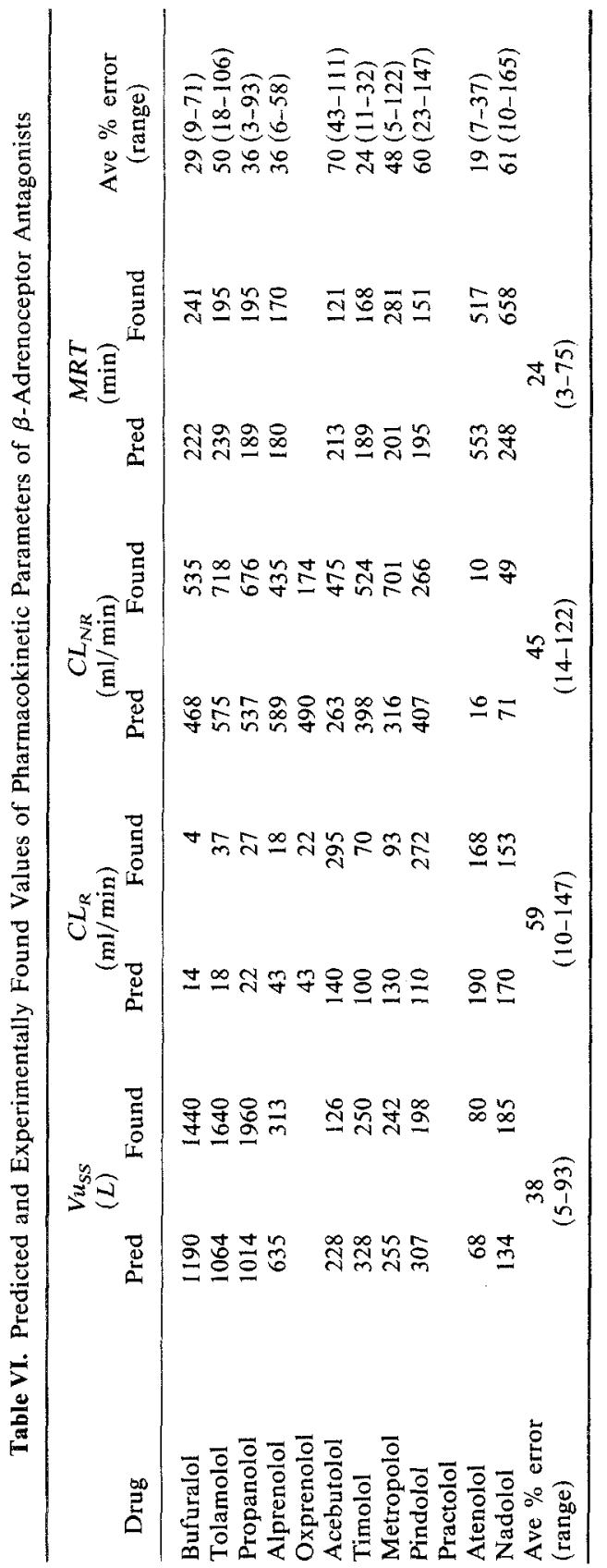




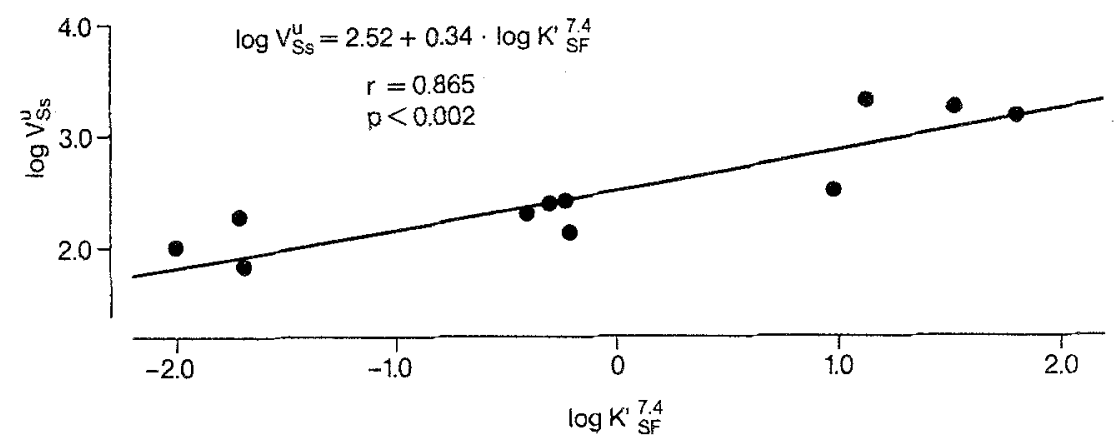

Fig. 4. Correlation between the steady state volume of distribution referenced to the unbound drug in plasma and the octanol/buffer partition coefficient by the shake flask method.

$M R T$ based on the regressions of these parameters on $K_{S F}^{17.4}$ and $K p$ are given in Table VI.

It can be seen that the recalculated parameters for timolol no longer deviated systematically from the best fitting line in the regressions (Figs. 4, 8 ) as they did previously. Consequently, the recalculated mean percent errors of the predicted values for $V u_{s s}$ and $M R T$ were smaller, and were 38 and $24 \%$ as against the previously obtained 51 and $48 \%$, respectively (Table IV). The overall mean percent error for the predicted values of all the parameters considered also decreased from 60 to $42 \%$.

In summary, all the conclusions drawn previously for the series of BA tested could be confirmed: there existed significant structure-pharmacokinetic relationships for beta-adrenoceptor antagonists.

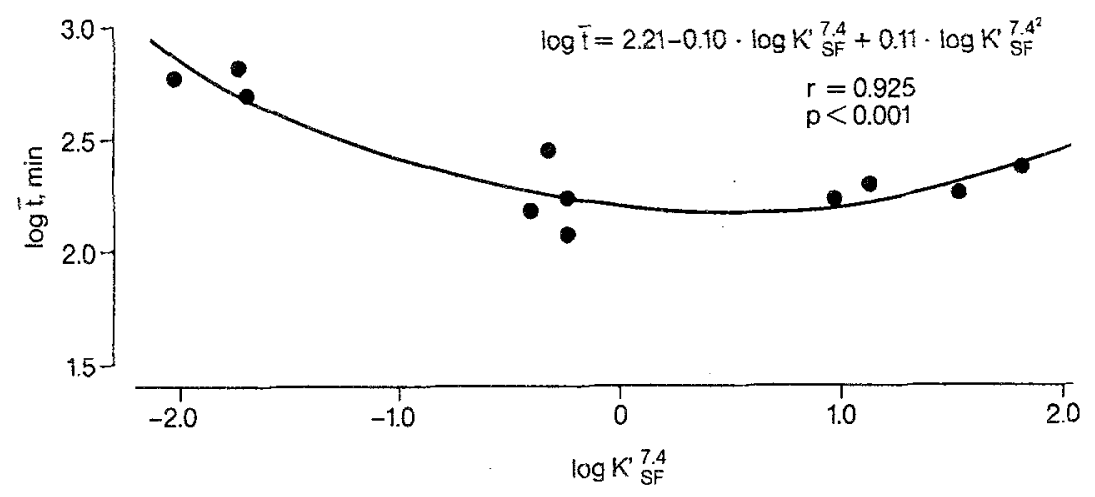

Fig. 8. Correlation between the mean residence time and the octanol/buffer partition coefficient by the shake flask method. 
The majority of the studied pharmacokinetic parameters depended on the apparent octanol/buffer ( $\mathrm{pH} 7.4)$ partition coefficient. Significant correlations existed also among the pharmacokinetic parameters tested. Lipophilicity/hydrophilicity was the major determinant controlling the pharmacokinetic behavior of all the compounds studied, including timolol, which previously deviated systematically from the other BA. This result for timolol was new. 